96 Zacharias: Momentum Dependency of the Volume Plasmon Damping in Metals

Especially the observation of a hump in the $\Delta E_{1 / 2}(k)$-curve needs a theoretical explanation. Perhaps at certain $k$-values there exists a larger probability for oblique interband transitions or for absorption and emission of phonons. Further experimental and theoretical work is necessary.

This work has been carried out in the Institut für Angewandte Physik der Universität Hamburg and has been supported by the Deutsche Forschungsgemeinschaft. The author would like to thank Prof. Dr. H. Raether for his stimulating interest and valuable discussions.

Dipl.-Phys. Peter Zacharias Institut für Angewandte Physik der Universität Hamburg D-2000 Hamburg 36, Jungiusstr. 11 Federal Republic of Germany

\title{
Erratum
}

\section{Influence of Atom-Atom Collisions on the Collisional-Radiative Ionization and Recombination Coefficients of Helium Plasmas}

\section{by H.W. Drawin and F. Emard}

Z. Physik 254, 202-217 (1972)

The paper contains some misprints.

In reaction (14): read $\mathrm{He}\left(1^{1} S\right)$ in stead of $\mathrm{H}_{e}\left(1^{1} S\right)$.

In Ref. [4]: read 45 in stead of 45.

On page 205 , heading of paragraph: read

in stead of

Collisional-Radiative Model; Rate Coefficients

$$
\text { Collisional-Radiative Model Rate, Coefficients }
$$

On page 212,17 th line from the bottom: read

... here have recently been ...

On page 215, first line below Eq. (31):

read $\left(k T_{a} / \pi \mu\right)^{\frac{1}{2}}$ in stead of $\left(k T_{a} / \pi \mu\right) \frac{1}{2}$

H. W. Drawin

F. Emard

Association EURATOM-CEA

Fontenay-aux-Roses/France 